\title{
Gestión de colecciones en entornos consorciados: modelos de catálogos colectivos y organización de recursos electrónicos
} Artículo

\author{
Por Gisela Ruiz Chacón
}

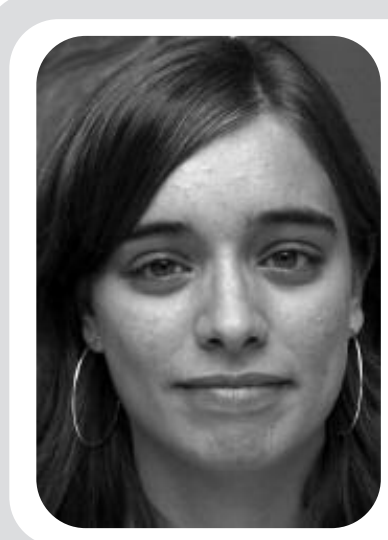

Diplomada en biblioteconomía y documentación y estudiante de la licenciatura en documentación, ha trabajado principalmente en bibliotecas universitarias (Universitat de Barcelona-Biblioteca de Dret, biblioteca de Esade) y especializadas (biblioteca del International Peace Research Institute (Oslo)). Ha trabajado también en la linea de automatización de la empresa GreenData, como product manager de los productos de Ex Libris (Aleph, MetaLib, SFX, DigiTool y Verde).

Resumen: Los consorcios bibliotecarios han tenido mucha importancia en la mejora de los servicios ofrecidos por las bibliotecas que forman parte de ellos. Estas instituciones han potenciado la aceptación y el uso de estándares y formatos que garantizan el intercambio de datos, han apoyado desarrollos específicos para solucionar las necesidades de sus usuarios, y, por encima de todo, han influido de manera determinante en la racionalización de los recursos económicos y en la mejora de la gestión de las colecciones. El artículo se centra en dos de los principales impactos actuales de los consorcios: los modelos de catálogos colectivos y la gestión consorciada de recursos electrónicos. La consolidación de nuevos sistemas en los próximos años cambiará el rumbo de las bibliotecas porque supondrán un avance en la gestión interna de los recursos electrónicos y un cambio radical en la presentación de las colecciones al usuario.

Palabras clave: Catálogos colectivos, Consorcios de bibliotecas, Recursos electrónicos, Gestión de colecciones, Sistemas de gestión de bibliotecas, Cooperación bibliotecaria, Bibliotecas híbridas, Portales bibliotecarios.

\section{Title: Managing library collections in consortia environments: union catalogue models and e-resource management.}

\begin{abstract}
Library consortia have been very important in enhancing library services offered to patrons. These institutions have promoted the acceptance and use of library standards and formats, thus guaranteeing data exchange between libraries. Library consortia also have supported specific technological developments to solve patron needs and, more importantly, have played a very important role in managing economic resources and in improving collection management. This article discusses the impact of library consortia by concentrating on two main aspects: union catalogues and e-resource management within a consortia environment. The consolidation of new library systems in the coming years will change the course of libraries by allowing progress to be made on internal management of e-resources and by altering the way in which collections are presented to patrons.
\end{abstract}

Keywords: Union catalogues, Library consortia, Electronic resources, Collection management, Automated library systems, Hybrid libraries, Library portals, Library co-operation.

Ruiz Chacón, Gisela. "Gestión de colecciones en entornos consorciados: modelos de catálogos colectivos y organización consorciada de recursos electrónicos”. En: El profesional de la información, 2005, mayo-junio, v. 14, n. 3, pp. 174-189.

\section{Introducción: cooperación bibliotecaria}

Se puede definir como "un sistema de recursos compartidos que consiste en una serie de actividades bibliotecarias mediante las cuales cada participante aporta algo útil para los demás y recibe algo útil a cambio" (Voigt, 1977). Dicha cooperación implica que los miembros que participan trabajen conjuntamente para conseguir un fin, minimizando costes y maximizando resultados. Así, Ramírez Escárcega (1983) la define como "la ayuda mutua que se brindan y reciben entre dos o más bibliotecas a fin de satisfacer las necesidades de información de sus usuarios y hacer un uso más racional de sus recursos".

Los primeros casos de cooperación entre bibliotecas se remontan al siglo XIII cuando 138 bibliotecas 
monásticas de Inglaterra y Escocia se unieron para crear un catálogo colectivo conocido como Registrum librorum Angliae. No es hasta el siglo XX cuando la cooperación bibliotecaria adquiere mayor fuerza. En la década de los 30, la University of North California y la Duke University unieron sus esfuerzos para incrementar las colecciones que tenían disponibles, centrándose cada una en un área concreta del conocimiento. Fueron pioneras en el movimiento cooperativo que se produciría a finales del siglo XX.

Para hablar de "cooperación bibliotecaria" tal y como la entendemos hoy en día, es necesaria una serie de elementos que según Merlo (1999) se condensan en los siguientes:

-Conjunto de bibliotecas que compartan información, servicios, etc.

-Acuerdos que formalicen la colaboración.

- Normas para llevar a cabo las actividades.

-Estructura organizativa donde estén representadas todas las bibliotecas.

-Infraestructuras y medios técnicos.

-Objetivos, programas y proyectos.

Así pues, la definición que nos ofrece Merlo acota el concepto de "cooperación bibliotecaria" y nos permite ver sus límites y sus posibilidades: "actividades llevadas a cabo por varias bibliotecas a partir de acuerdos, de manera conjunta y normalizada, persiguiendo objetivos comunes y poniendo al servicio de la colaboración el personal y los medios necesarios".

\section{Webs consultados*}

- Endeavor http://www.endinfosys.com

- Ex Libris http://www.exlibrisgroup.com

- Innovative Interfaces http://www.iii.com

- Muse Global http://www.museglobal.com

- Sirsi http://www.sirsi.com

- VTLS http://www.vtls.com

- WebFeat http://www.webfeat.org

*Todos en la fecha II-04-05
Aunque se considera que la cooperación bibliotecaria (al menos como la entendemos en nuestros días) tuvo sus inicios en la década de los 60 con la automatización de las bibliotecas, hubo que esperar hasta mediados de los 90 para que estallara el boom. Dicha cooperación se hizo especialmente visible en entornos académicos y de investigación, propiciando la aparición de los primeros consorcios de bibliotecas. Sus inicios estuvieron marcados por un contexto económico restrictivo, una clara voluntad política y de solidaridad bibliotecaria, así como unos importantes avances tecnológicos que facilitaron el intercambio y la comunicación entre bibliotecas.

\section{«Los consorcios han potenciado la aceptación y el uso de estándares y formatos bibliotecarios, han apoyado desarrollos tecnológicos específicos y han influido en la racionalización de los recursos y la mejora de gestión de las colecciones»}

Los consorcios bibliotecarios aparecieron para reafirmar la voluntad cooperativa de las bibliotecas y reforzar las alianzas entre centros dotando de unos objetivos, una estructura y unos procedimientos a acciones cooperativas puntuales.

El movimiento de creación de consorcios fue extendiéndose por los cinco continentes, posibilitando la formación de gran cantidad de redes bibliotecarias en todos los países, con estructuras muy diversas. Si hacemos un repaso a los consorcios existentes actualmente podemos constatar esta variedad y clasificarlos según los parámetros que tomemos de referencia.

\section{Tipos de consorcios}

Según la clase de bibliotecas podemos encontrar consorcios de bibliotecas públicas, universitarias, especializadas y consorcios "híbridos" (entendiendo como tales los que incluyen bibliotecas de distinta tipología: nacionales, universitarias, públicas, etc.).

Según el alcance geográfico tenemos consorcios nacionales y regionales (especialmente importantes en Europa y EUA). En esta línea, Giordano (2002) destaca tres modelos emergentes:

-Nacional centralizado: la biblioteca nacional del país actúa como su eje principal y toma importantes decisiones que afectan al modus operandi de las bibliotecas y, en general, a la política bibliotecaria del país. 
-Nacional descentralizado: en este caso, distintos centros de un mismo país se unen para llevar a cabo acciones cooperativas sin la existencia de una biblioteca nacional que juegue un papel central o de políticas nacionales en materia bibliotecaria.

- Regional: formado por bibliotecas que pertenecen a "una zona territorial regulada por un sistema federal o descentralizado".

Según la estructura jurídica encontramos consorcios "de jure" (que disponen un estatus legal que les permite actuar como organización de servicios) y consorcios "de facto" (que cooperan entre ellos sin disponer de una base legal y en los cuales las relaciones son informales).

Por último, Giordano (2002) destaca un cuarto tipo determinado por el "origen de creación". Así pues, se diferencia de los creados para compartir recursos (a nivel de catalogación y de adquisiciones) y de los que se marcan como objetivo realizar proyectos de gestión consorciada de los recursos electrónicos.

\section{Influencia de los consorcios en las colecciones}

Los consorcios han potenciado la aceptación y el uso de estándares y formatos que garantizan el intercambio de datos, han apoyado desarrollos específicos para solucionar las necesidades de sus usuarios y, por encima de todo, han influido de manera determinante en la racionalización de los medios económicos y en la mejora de la gestión de las colecciones.

En este sentido, Anglada (2003) afirma que "los tres impactos principales de un consorcio sobre las colecciones son el catálogo colectivo, los mecanismos de préstamo y las compras conjuntas [especialmente de recursos electrónicos]".

A continuación nos centraremos en el impacto de los consorcios en las colecciones a dos niveles: los catálogos colectivos y la gestión consorciada de los recursos electrónicos que se plantea como consecuencia de las compras conjuntas.

3.1. Catálogos colectivos: modelos, aproximación y experiencias

Entendemos por catálogo colectivo un catálogo bibliográfico que contiene fondos de dos o más bibliotecas cuya finalidad es ser utilizado como herramienta para localizar obras que están disponibles en distintos centros. Además permite que las bibliotecas que lo constituyen compartan información sobre los usuarios y ofrezcan servicios conjuntos a éstos, respetando la autonomía y la independencia de cada una de ellas.
Los sistemas de gestión bibliotecaria actuales incorporan módulos específicos para los catálogos colectivos, así como para entornos consorciados, ofreciendo soluciones variadas en función de las necesidades y la estructura de los mismos. Además facilitan su creación a partir de catálogos locales funcionando con distintos sistemas de gestión bibliotecaria, logrando una total integración de recursos y acabando con el estigma del productor. Ahora es posible la cooperación más allá del sistema informático elegido.

\section{«El modelo de catálogo colectivo tradicional se construye sobre una base de datos centralizada fruto de la unión física de diversos catálogos individuales»}

Si observamos el panorama bibliotecario actual repararemos en que hay varios modelos de catálogos colectivos desarrollados específicamente en base a las necesidades de cada biblioteca. Entre los más destacados encontramos: el tradicional, el de visualización única, el central y el virtual.

\subsubsection{Modelo de catálogo colectivo tradicional}

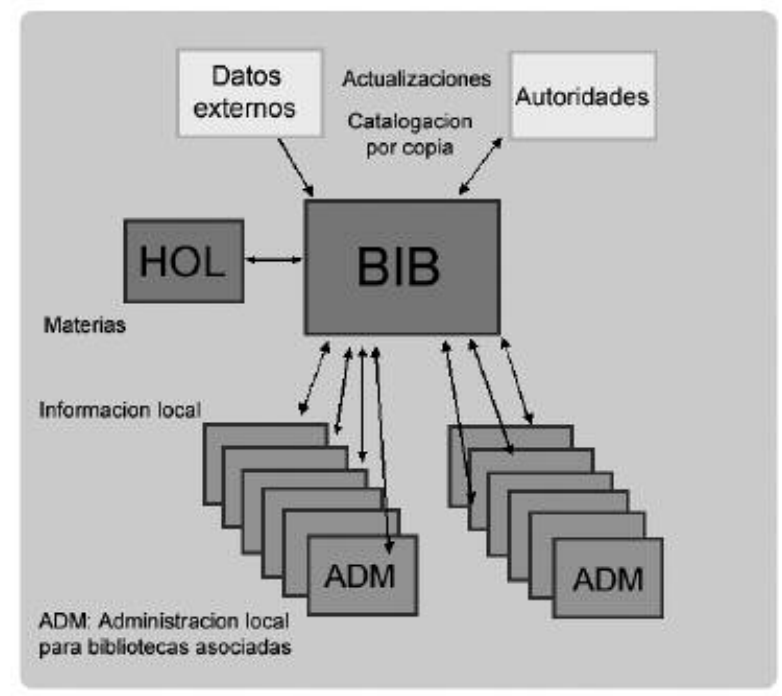

\section{Modelo: CATÁLOGO COLECTIVO}

Se construye sobre una base de datos centralizada fruto de la unión física de diversos catálogos individuales y se caracteriza por:

—Estar constituido por registros bibliográficos independientes.

- Contener información sobre notas y fondos locales de cada biblioteca.

-Disponer de tablas preconstruidas de equivalencia de registros que reducen el tiempo de respuesta del 
sistema y agilizan los procesos de desduplicación y fusión de resultados.

- Mostrar los resultados desduplicados y fusionados en el web opac, en el servidor Z39.50 y en el servidor $\mathrm{xml}$.

-Acceder en tiempo real al estado de circulación de los documentos vía Z39.50 u otros protocolos.

- Garantizar tablas de configuración compartidas e índices comunes entre los distintos miembros que constituyen el catálogo.

Un ejemplo de este tipo es Melvyl $^{l}$ cuyo diseño y arquitectura se basan en el reconocimiento de diferentes sistemas de gestión bibliotecaria. Es el catálogo colectivo de las bibliotecas de campus de la University of California, California State Library, Hastings College of the Law, California Academy of Sciences, California Historical Society, Center for Research Libraries, Graduate Theological Union, y Lawrence Berkeley National Laboratory. En 2003 fue reemplazado por un nuevo catálogo colectivo basado en Aleph 500 que se desarrolló conjuntamente con la California Digital Library $^{2}$ y Ex Libris. Contiene 23 millones de títulos que en el momento de la recuperación de la información se desduplican y se fusionan para la visualización.

\section{Catálogo MELVYL}

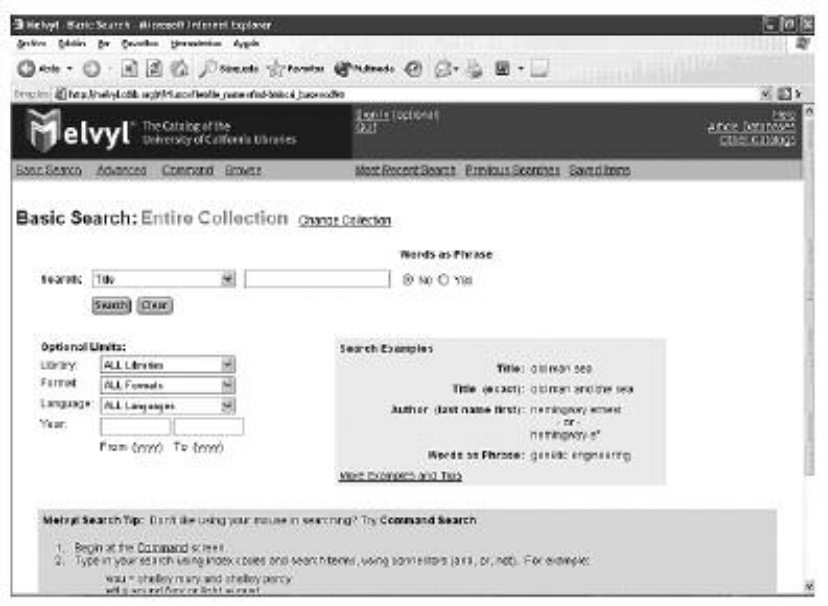

Modelo: catálogo colectivo

La independencia de los catálogos locales de cada una de las instituciones y de los diferentes campus ha sido, y es, una de las exigencias principales por parte de los miembros del consorcio para crear el catálogo colectivo. Por este motivo los registros bibliográficos no se fusionan físicamente sino que lo hacen dinámicamente en la visualización. De esta manera los usuarios finales pueden ver tanto el registro global como el local de un campus en su propio web opac y tener acceso al estado de circulación en tiempo real. Una gran variedad de parámetros, un control de las tablas de configuración, una herramienta para desduplicar registros a tiempo real y una constitución de grupos predefinidos de registros equivalentes han mejorado mucho su rendimiento.

\subsubsection{Modelo de visualización única}

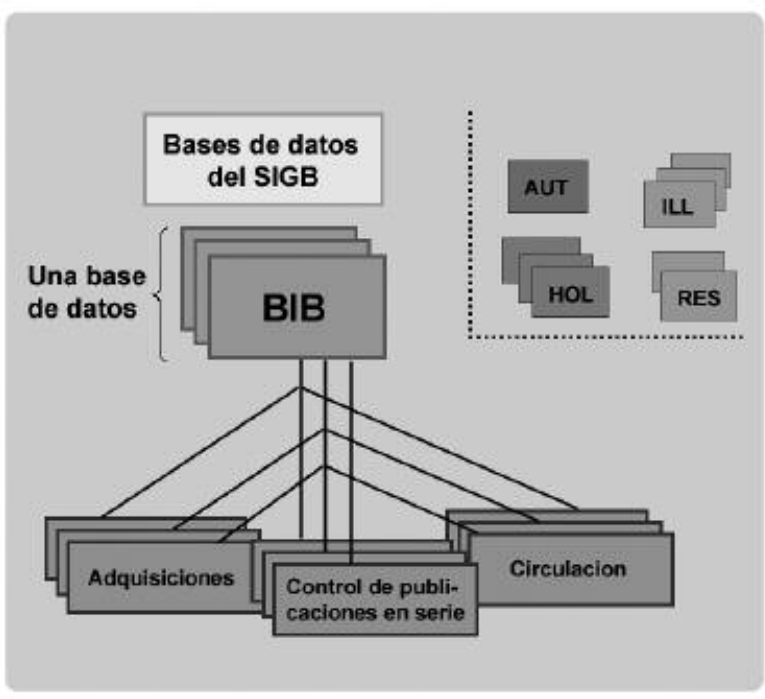

Modelo: visualización única

Es una variación del anterior y coinciden en que la base de datos central diferencia los registros de cada una de las instituciones miembro del consorcio, aunque se trata de una única base de datos bibliográfica compartida. La visualización única se presenta como una base de datos lógica y se materializa en el web opac y en el servidor Z39.50.

\section{«El modelo de visualización única dispone de una base de datos central que diferencia la procedencia de los registros aún siendo la misma base de datos bibliográfica»}

Sus características principales son:

—existencia de registros bibliográficos separados,

- procesos independientes de adquisiciones, control de publicaciones periódicas y circulación,

- resultados desduplicados para el usuario final,

- visualización fusionada e integrada de registros $\mathrm{y}$ fondos locales en el web opac, y

- visualización de los registros separados en la interfaz GUI del personal bibliotecario.

Un ejemplo de este modelo está en la City University of New York $(C U N Y)^{3}$, cuya biblioteca se encuentra formada por 19 bibliotecas de campus, cada una de 
las cuales tiene su catálogo con sus propios fondos. Aunque se trate de una biblioteca central con sucursales es un ejemplo muy interesante puesto que cada una de ellas es completamente independiente de las demás y han optado por un modelo de catálogo colectivo frente a la utilización de un único opac con localizaciones diferenciadas. El catálogo colectivo de $C U N Y$ se basa en Aleph 500 y se implementó en el año 2001. Antes de la migración al nuevo sistema, su catálogo contenía registros separados para cada uno de los títulos mantenidos. Con el modelo de visualización única todos los registros bibliográficos separados y subyacentes se unificaron para su uso público, creando un único catálogo de fondos bibliográficos para los usuarios finales y garantizando un rendimiento óptimo del sistema.

\section{Catálogo CUNY}

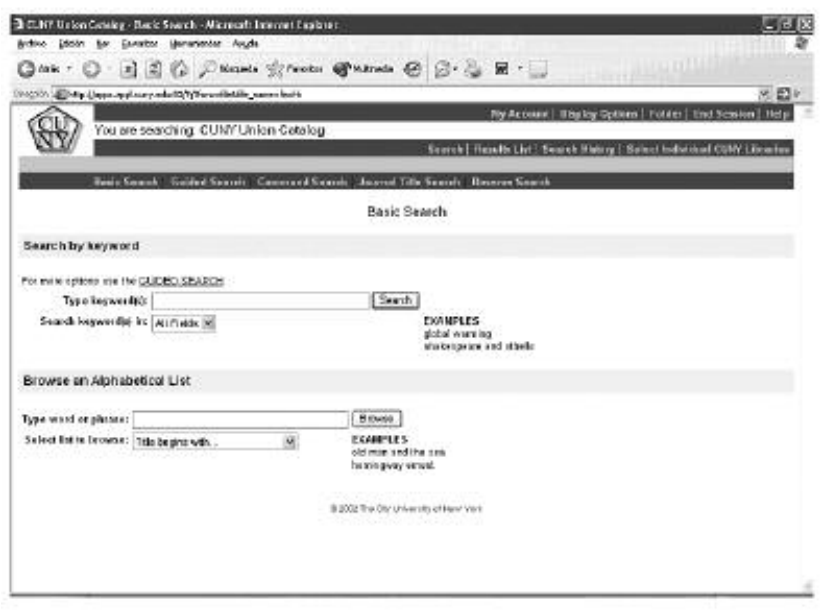

Modelo: visualización única

Otro ejemplo más cercano lo encontramos en el catálogo colectivo Rebiun ${ }^{4}$ (Red de Bibliotecas Universitarias Españolas), puesto en línea el 1 de julio de 2000 y que contiene los fondos de las bibliotecas universitarias del estado, el Consejo Superior de Investigaciones Científicas, el Consorci de Biblioteques Universitàries de Catalunya (CBUC) y la Red Universitaria Española de Catálogos Absys (Rueca). En realidad se trata de tres catálogos colectivos: el catálogo completo, el catálogo de publicaciones periódicas y el catálogo de fondo antiguo basados en AbsysNET. Cada biblioteca miembro cataloga sus propios recursos y periódicamente, cada seis meses, se hace la actualización del catálogo colectivo. La consulta se realiza mediante el opac de AbsysNET y el servidor Z39.50. A nivel de desduplicación existen algoritmos que se encargan de determinar qué registros son equivalentes para mostrarlos de manera única al usuario. La localización de los fondos se queda a un nivel básico puesto que únicamente indica en qué institución se encuentra el do- cumento recuperado (da los datos de la biblioteca y el acceso general al catálogo pero no la localización exacta o el enlace concreto a ésta).

\subsubsection{Modelo de catálogo colectivo central}

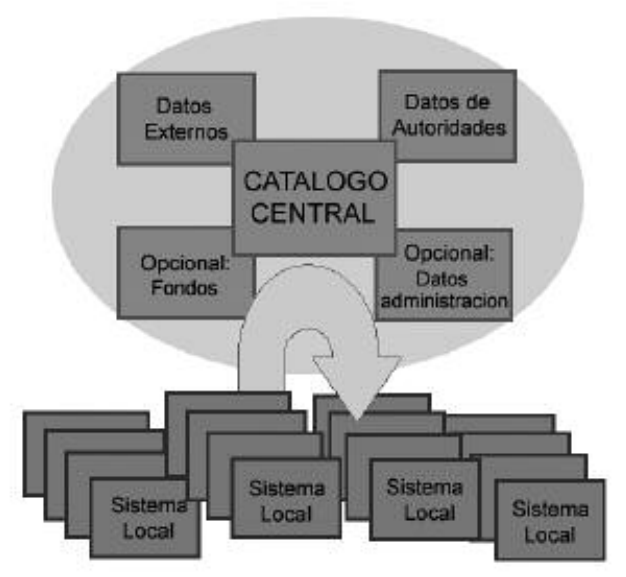

\section{Modelo: catálogo colectivo central}

Está destinado a grandes consorcios y se basa en un catálogo central complementado por los catálogos locales de las bibliotecas miembros. Además incorpora una funcionalidad automática de actualización de los catálogos: cuando se actualiza un registro bibliográfico, un servicio central revisa los cambios o incorporaciones en el catálogo central. A su vez existe otro servicio en la biblioteca local que hace un seguimiento de los cambios producidos en el catálogo central. Un tercer servicio se encarga de actualizar los catálogos locales en base a las modificaciones del central.

Entre las principales características de este modelo destacan:

- Actualizaciones de registros de la base de datos central a las bibliotecas locales.

-Fusión en local de los registros bibliográficos centrales y locales en función de las necesidades de cada biblioteca.

-Actualización de fondos centrales a través de los fondos locales.

-Servidores centrales y locales.

Originalmente este modelo fue implementado en 57 bibliotecas miembros de la Austrian Library Network ${ }^{5}$, el consorcio de bibliotecas universitarias, administrativas y de investigación más grande de Austria. Todas ellas comparten un catálogo central del cual cada una extrae los registros que son de su interés para uso local. Cualquier actualización del catálogo central se refleja automáticamente en todos los locales gracias 
La próxima generación de Enlaces Universal Acceso al lexto complete, recursos web y bases de datos con un cli zo del ratón: LinkSolver de Ovid arganiza el mundo de informpción a su alcance

\section{Lanzamiento} inminente: Funcionalidades de Metabuscador 
a un módulo especial del sistema de gestión de bibliotecas que utilizan y que facilita esta acción.

\section{Catálogo Austrian Library Network}

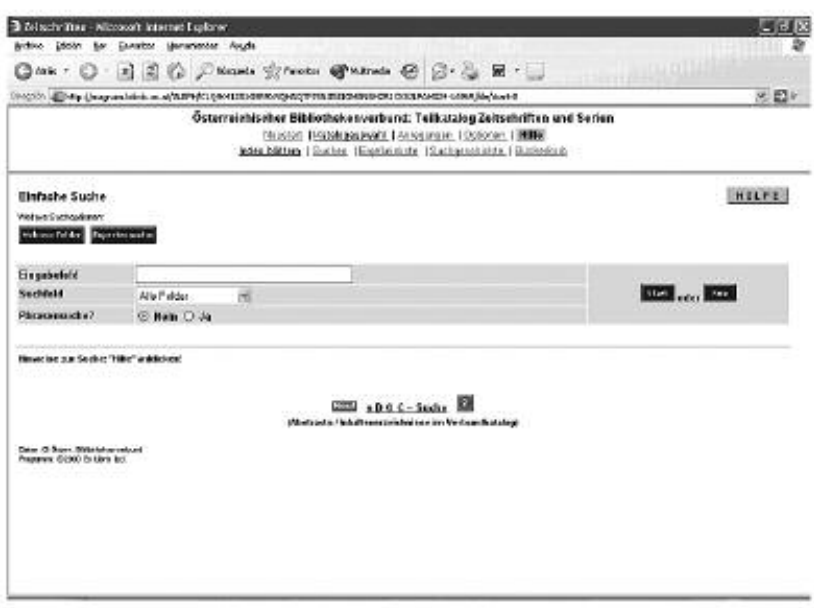

Modelo: catálogo colectivo central

HBZ (Hochschulbibliothekszentrum des Landes Nordrhein-Westfalen), State University Service Center es uno de los catálogos bibliográficos más grandes de Europa. Las bibliotecas que forman parte de él utilizan sistemas de gestión bibliotecaria distintos y unen sus fondos en un catálogo colectivo que sigue el modelo central.

\section{«El modelo de catálogo colectivo central se basa en un catálogo central complementado por catálogos locales»}

El CCUC $C^{6}$, catálogo colectivo del Consorci de Biblioteques Universitàries de Catalunya $(C B U C)$ sigue también este patrón. Contiene los fondos de la Biblioteca de Catalunya, las universidades catalanas y otras instituciones asociadas que colaboran en su alimentación. Cada vez que se quiere añadir un registro hay que consultar si ya existe en el catálogo central. En caso de que así sea, la biblioteca añadirá los fondos locales y descargará el registro a su catálogo local. En caso contrario lo creará, catalogará el documento, añadirá los fondos y lo copiará a su catálogo.

\subsubsection{Modelo de catálogo colectivo virtual}

Está orientado a bases de datos distribuidas en catálogos locales conectadas vía internet. La catalogación se lleva a cabo en los sistemas locales y la información se actualiza en el catálogo central. De este modo cada biblioteca mantiene un catálogo virtual consultable gracias a un índice compartido.
Este modelo se desarrolló en un primer momento para el KOBV (Kooperativer Bibliotheksverbund Berlin-Brandenburg), la red de bibliotecas cooperativas de los estados federados de Alemania, Berlín y Brandenburgo. Se basó en las necesidades del consorcio para establecer unos servicios centrales para todas las bibliotecas (p. ej: uso común de autoridades) y crear un catálogo consultable vía internet a través de un índice compartido. Esta innovadora red bibliotecaria está compuesta por distintas bases de datos distribuidas en catálogos locales independientes y conectadas a través de la Red que se comunican entre ellas mediante protocolos internacionales como Z39.50, http o utilizando xml. La catalogación se lleva a cabo desde los sistemas locales actualizando el catálogo central de abajo hacia arriba.

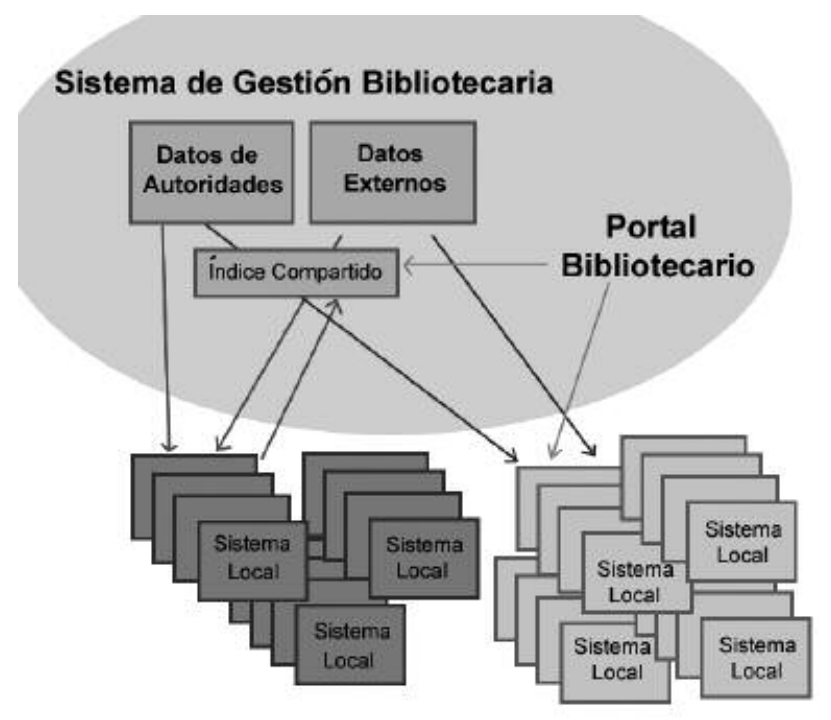

Modelo: catálogo colectivo virtual

Este modelo virtual renuncia a un catálogo colectivo tradicional en favor de un almacenamiento distribuido de datos y la catalogación principal en un sistema local basado en bases de datos de autoridades y recursos externos.

\section{«El catálogo colectivo virtual está orientado a bases de datos distribuidas en catálogos locales conectados vía internet»}

Las principales características del catálogo colectivo virtual son:

—Compatibilidad con http, Z39.50 y xml.

-Posibilidades búsqueda a texto completo y a índices específicos desde el opac.

—Distribución de las peticiones a las instituciones locales. 

os.

-Desduplicación y normalización de los resulta-

-Funcionalidades de reserva y entrega de documentos.

-Creación de perfiles de búsqueda.

—Disponibilidad de bases de datos de autoridades y recursos externos.

—Acceso a datos no bibliográficos.

—Servicios de administración y evaluación del sistema.

\section{Catálogo KOBV}

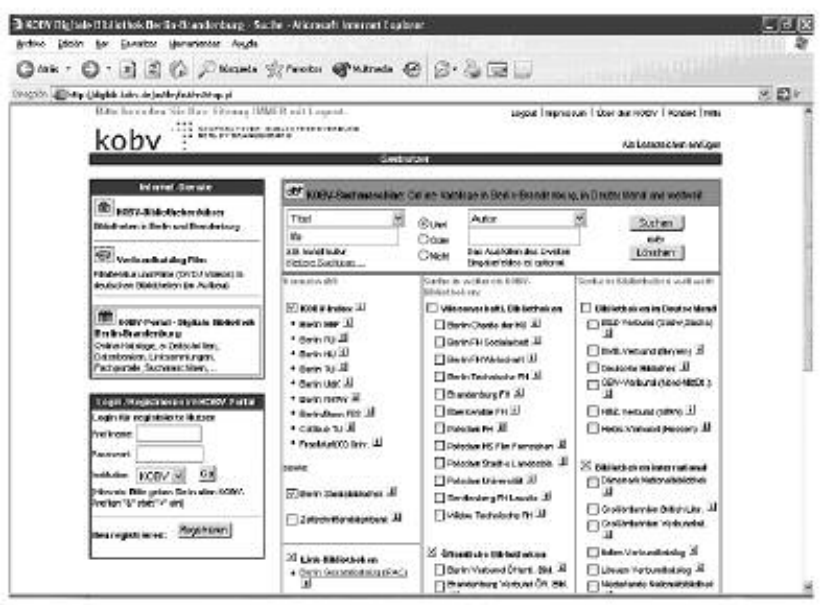

Modelo: catálogo colectivo virtual

Otra característica importante es que el web opac provee resultados de la búsqueda basados en los índices seleccionados, desduplica resultados y proporciona servicios de reserva y entrega de documentos para los usuarios de los centros que configuran el consorcio. Todos los usuarios pueden configurar libremente sus perfiles individuales y personalizar su entorno de trabajo adaptándolo a sus necesidades.

\subsection{Gestión consorciada de recursos electró- nicos}

Las compras conjuntas suponen la adquisición de objetos (revistas-e, bases de datos, etc.) o, en la mayoría de los casos, la autorización de acceso a la información electrónica deseada. Estas compras repercuten positivamente en las bibliotecas consorciadas por dos motivos principales:

-implican un incremento neto de las colecciones, y

- suponen ahorros o contenciones en los precios (Anglada, 2003).

Hay que tener en cuenta que estas compras (que solucionan dos problemas tan importantes como el aumento de las colecciones y el ahorro de costes) plante- an un nuevo reto: la gestión y la puesta a disposición de los usuarios de estos activos adquiridos de manera compartida entre distintas instituciones.

En esta línea se han desarrollado potentes soluciones conocidas como "library portals" o "resource discovery tools" que ponen fin a la situación actual que se da en entornos bibliotecarios consorciados y facilitan la integración de las fuentes físicas y electrónicas.

Los "library portals" son herramientas constituidas por dos componentes diferenciados: la interfaz del usuario y la del bibliotecario. La primera permite presentar los recursos de la biblioteca de manera estructurada y ofrece herramientas de búsqueda y recuperación de la información en toda la colección. Con la segunda, los bibliotecarios describen y estructuran los contenidos (revistas electrónicas, bases de datos, e-books, opacs, etc.) que serán mostrados en la interfaz del usuario.

\section{Portal bibliotecario Universidad de Maryland}

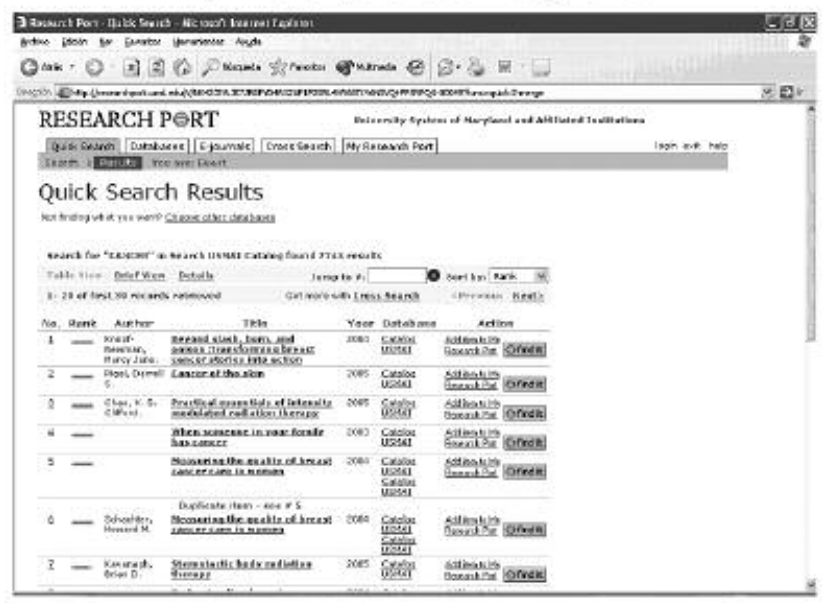

\section{Integración recursos electrónicos y físicos}

Hay que diferenciar entre "library portals" y meros buscadores transversales puesto que suele haber confusión. Un "library portal" se caracteriza por disponer de:

—Un módulo de gestión de recursos electrónicos tanto a nivel de catalogación como de difusión.

—Un módulo de gestión de usuarios y de licencias de acceso a los recursos electrónicos.

-Un metabuscador.

—Entornos personalizados para los usuarios.

—Un módulo de estadísticas de uso.

Estos productos permiten un control detallado de las licencias de acceso determinando grupos de usuarios a distintos niveles (universidad, facultad, tipología de usuarios, etc.) con permisos para acceder a materiales concretos. El usuario final tendrá acceso transpa- 
rente a todas las suscripciones que la biblioteca pone a su disposición a través de una única interfaz que no diferencia entre títulos contratados por el consorcio o por la propia biblioteca.

Habitualmente los portales bibliotecarios se complementan con los llamados servidores de enlaces, los cuales por norma general se basan en el estándar Open-URL y tiene como finalidad interrelacionar toda la colección. De esta manera el usuario puede obtener enlaces sensibles al contexto de búsqueda en función de los fondos que tenga disponibles la biblioteca y la consulta que haya realizado. El tipo de enlace o servicios que suelen ofrecer estos servidores son el acceso al texto completo, la consulta de publicaciones en el catálogo local, la petición de préstamos interbibliotecarios, ampliar la consulta, etc.

Por ejemplo, imaginemos que un usuario está consultando la base de datos PubMed y que recupera un conjunto de registros bibliográficos que son de su interés. Un servidor de enlaces le permitiría acceder al texto completo en $B M J$ (si se hallara dentro de los fondos de su biblioteca), realizar una petición de préstamo interbibliotecario a la British Library, obtener el factor de impacto de las revistas cuyos artículos ha recuperado a través de ISI Journal Citation Report, guardar las referencias bibliográficas en un gestor de citas (ej. EndNote, ProCite, Reference Manager, RefWorks, etc.) y obtener la OpenURL de los artículos.

En el mercado hay muchos ejemplos de portales bibliotecarios y servidores de enlaces creados por empresas tradicionalmente dedicadas al desarrollo de software bibliotecario. A continuación haremos una relación de los más destacados a nivel nacional e internacional.

\section{—MetaLib \& SFX (Ex Libris).}

La solución de Ex Libris incorpora múltiples herramientas y funcionalidades, destacando como una de las más completas en el mercado. Dispone de 1) un metabuscador que reproduce las consultas del usuario a todos los recursos contratados por la biblioteca; 2) un módulo de catalogación de los recursos electrónicos (ya sean bases de datos, catálogos, etc.); 3) un módulo de registro de usuarios que permite diferenciar usuarios autorizados e invitados para controlar los derechos de propiedad intelectual; 4) servicios de personalización para el usuario y 5) un módulo de estadísticas. Además, viene conjuntamente con $S F X$, el servidor de enlaces de Ex Libris ambos productos son completamente multilingüe.

\section{_EnCompass \& LinkFinder.}

Producto de la empresa Endeavor que se basa en tecnología de MuseGlobal. Ofrece herramientas para la gestión de la biblioteca digital e incluye un metabuscador que realiza consultas a través de distintos protocolos (http, xml o Z39.50). Permite gestionar de manera conjunta colecciones locales, recursos web gratuitos y de pago (bases de datos y revistas electrónicas). La versión básica facilita el acceso al texto completo de los artículos y a sus citas bibliográficas a través de LinkFinder, su servidor de enlaces. La versión avanzada incluye LinkFinderPlus que brinda crear vínculos adicionales a bases de datos y motores de búsqueda, así como obtener información extra sobre recursos locales y externos.

\section{—Millenium Access Plus (MAP).}

La casa Innovative Interfaces ofrece MAP como un módulo adicional a Millenium, su sistema de gestión bibliotecaria. Está formado por tres elementos: MetaFinder (metabuscador), WebBridge (servidor de enlaces) y WAM (servidor proxy para la autentificación remota de usuarios). Se basa en tecnología de $M u$ seGlobal y su función principal es la metaconsulta en recursos a texto completo, bases de datos, catálogos de bibliotecas, motores de búsqueda, etc. Aporta escasas herramientas para la organización y la presentación de los recursos.

\section{—Sirsi SingleSearch de Sirsi.}

Es el metabuscador de Sirsi basado también en tecnología MuseGlobal. Tiene funcionalidades de personalización, autentificación y estadísticas y se integra en distintos productos de Sirsi (iBistro, iLink, Web2 y Sirsi Rooms) mejorando sus capacidades de recuperación de información.

\section{-Vectors.}

Producto de VTLS que se ofrece como módulo adicional a Virtua, el nuevo sistema de gestión bibliotecaria de la empresa americana. La interfaz de búsqueda es muy similar a la de un opac y posibilita al usuario localizar información en sistemas Z39.50 y también en otros que no se basen en este protocolo. Ofrece filtros tradicionales de búsqueda (formato, fecha, etc.) y almacenamiento del historial de búsquedas durante una sesión.

\section{-MuseSearch.}

La solución de MuseGlobal se basa en una tecnología abierta que puede trabajar simultáneamente con distintos productos puesto que traduce sus protocolos externos a formato xml para poder gestionar y presentar los resultados de cara al usuario. En esencia no se trata de un portal bibliotecario pero Muse ha diseñado herramientas adicionales que hacen su producto tan competitivo como los de las empresas tradicionalmente dedicadas a la venta de software bibliotecario. 
MuseGlobal ha cedido su tecnología a distintas empresas: Innovative Interfaces, Endeavor, Sirsi, TLC, etc.

\section{-WebFeat.}

Este portal se caracteriza por realizar la búsqueda virtual en cualquier fuente de información que se halle en internet, facilitar la autentificación de usuarios y potenciar la personalización de entornos. Un ejemplo claro de su implementación lo encontramos en Web of Knowledge, de ISI.

Existen otros productos en el mercado que, aunque minoritarios, merecen ser mencionados: AGent (Auto-Graphics), Zones (BiblioMondo), Horizon Information Portal (Dynix), ZPortal (Fretwell-Downing), Polaris 3.0 (Gaylord), YouSeeMore $(T L C)$, entre otros. Incluso los agregadores de contenidos han empezado ha desarrollar sus propias soluciones: Serials Solutions de ProQuest y LinkSolver de Ovid $^{7}$ son dos ejemplos claros.

Con tanta oferta ¿cómo diferenciamos estos productos? En primer lugar hay que distinguir entre aquellos que sólo son servidores de enlaces, los que son meros buscadores transversales y los que entran en la categoría de "library portals". Hay numerosos informes y artículos que tratan dicha cuestión -Dorner, Curtis, 2003; Jacsó, 2004; Ferguson, Grogg, 2004- pero las diferencias básicas radican en la manera cómo se realiza la búsqueda, cómo se recuperan los resultados y en las herramientas adicionales que incorporan al metabuscador: listados dinámicos de recursos, módulo de catalogación, personalización, funcionalidades de valor añadido para el usuario y para el bibliotecario, etc.

Chvatal (2003), en el informe de la ALA Midwinter, establece 11 aspectos que deben tenerse en cuenta cuando se evalúan sistemas de estas características:

1. Personalización de la interfaz de usuario. Las empresas de software ofrecen paquetes estándar de sus productos en los que esta característica es igual para todos los clientes. Hay que valorar la manejabilidad y capacidad de personalización de las interfaces para poder asemejarlas a la imagen corporativa de la institu- ción, así como introducir logotipos, cambiar funcionalidades, redistribuir el espacio o elegir colores, formatos y tipografías.

2. Búsqueda. Evaluar las funcionalidades de consulta es una de las tareas más complejas. Hay que tener en cuenta:

a. Interfaces de búsqueda. El portal debe proporcionar una interfaz unificada para todos los recursos, así como enlaces a las interfaces nativas para que los usuarios puedan consultarlas directamente. También tiene que incluir una de naturaleza simple para usuarios noveles y otra avanzada para los más expertos.

b. Búsqueda transversal. Capacidad de buscar de manera simultánea en distintos recursos a la vez. Es importante estudiar los protocolos utilizados para lle- 
EnCompass MetaLib Vectors

BÚSQUEDA

¿Proporciona una interfaz de búsqueda unificada?

¿Porporciona enlaces a las interfaces de los distintos recursos ofrecidos por la biblioteca?

¿Porporciona una interfaz de búsqueda simple?

¿Porporciona una interfaz de búsqueda avanzada?

¿Permite la búqueda transversal en bases de datos, revistas electrónicas y OPACs?

¿Permite la búsqueda transversal en motores de búsqueda y webs en Internet?

¿Permite la búsqueda transversal por palabra clave tanto en citaciones bibliográficas como en el

texto completo?

¿Permite la búsqueda transversal por distintos campos (autor, título, materia, ISBN, año)?

¿Permite la interrogación de un único recurso a través de la interfaz del portal?

¿Permite el uso de operadores booleanos?

¿Permite el uso de truncamientos?

¿Permite el uso de operadores de proximidad?

¿Muestra los resultados por orden de relevancia?

¿Permite establecer límites de búsqueda en cualquier campo?

¿Permite presentar los resultados de manera ascendente y descendente?

¿Permite refinar las búsquedas a partir de los resultados obtenidos?

¿Se presentan en un listado único los resultados de los distintos recursos consultados?

¿Desduplica los resultados cuando en el listado fusionado?

\section{SERVICIOS PERSONALIZADOS}

$\begin{array}{llll} & \text { Ex Libris } & \text { VTLS } & \text { Interfaces } \\ & & \end{array}$

¿El usuario puede establecer sus preferencias de visualización?

¿El usuario puede establecer sus preferencias de búsqueda?

¿El usuario puede establecer alertas?

¿El usuario puede crear grupos de favoritos?

¿El usuario puede disponer de un historial de búsqueda?

¿El usuario puede decidir los campos a mostrar en la impresión?

¿El usuaario puede seleccionar los resultados que quiere imprimir o guardar?

¿El usuario puede descargarlos resultados a un gestor de referencias bibliográficas?

¿Permite imprimir los resultados?

TOTAL DE PUNTOS

\begin{tabular}{r|c|c|c|c|c} 
TOTAL DE PUNTOS & 32 & 32 & 24 & 23 & 32 \\
\hline DE LOS CRITERIOS CUMPLIDOS & $88,88 \%$ & $88,88 \%$ & $66,66 \%$ & $63,88 \%$ & $88,88 \%$
\end{tabular}

\section{AUTENTIFICACIÓN}

¿Permite la autentificación mediante dirección IP?

¿Permite la autentificación mediante usuario/password?

¿Permite la autentificación mediante nombre de dominio?

¿Permite la autentificación mediante LDAP?

¿Permite la autentificación mediante un servidor Proxy?

¿Permite la autentificación mediante Shibbolett?

TOTAL DE PUNTOS

$\%$ DE LOS CRITERIOS CUMPLIDOS

\begin{tabular}{|c|c|c|c|c|}
\hline$S$ & $S$ & $S$ & S & $S$ \\
\hline$S$ & $S$ & $S$ & $S$ & $S$ \\
\hline$S$ & $S$ & $S$ & $S$ & $S$ \\
\hline S & S & S & S & S \\
\hline$S$ & $S$ & $S$ & $S$ & $S$ \\
\hline S & S & $P$ & S & S \\
\hline$s$ & $S$ & $P$ & $S$ & $S$ \\
\hline$S$ & $S$ & $S$ & $S$ & $\mathrm{~S}$ \\
\hline S & S & S & S & S \\
\hline$S$ & $S$ & $S$ & $S$ & $S$ \\
\hline S & S & S & S & S \\
\hline$S$ & $\mathrm{~N}$ & $S$ & $S$ & $S$ \\
\hline$S$ & S & S & S & $S$ \\
\hline D & $\mathrm{N}$ & $S$ & $P$ & $S$ \\
\hline S & $S$ & $\mathrm{~N}$ & $P$ & $S$ \\
\hline$S$ & $S$ & $S$ & $S$ & $S$ \\
\hline$S$ & $S$ & $S$ & $S$ & $S$ \\
\hline$S$ & $S$ & $S$ & $S$ & $S$ \\
\hline 68 & 64 & 64 & 68 & 72 \\
\hline ,98\% & $86,49 \%$ & $86,49 \%$ & $91,98 \%$ & $100,00 \%$ \\
\hline
\end{tabular}

PROTOCOLOS DE COMUNICACIÓN

Z39.50

ERL

OpenURL

HTTP

MARC

DOI

SQL

EAD

TEI

XML

DublinCore

Protocolos propios

Otros protocolos

TOTAL DE PUNTOS

$\%$ DE LOS CRITERIOS CUMPLIDOS

SISTEMA

Windows

Macintosh

Linux

Otros Unix

Sun Solaris

\section{BASE DE DATOS DE RECURSOS PREDEFINIDOS}

\begin{tabular}{|r|c|c|}
\hline DE LOS CRITERIOS CUMPLIDOS & $84,61 \%$ & $\mathbf{9 2 , 3 1 \%}$ \\
\hline
\end{tabular}

\begin{tabular}{|c|c|c|}
\hline S & S & S \\
\hline S & S & S \\
S & F & S \\
N & N & S \\
\hline S & P & P \\
N & N & S \\
\hline S & S & S \\
N & S & P \\
\hline S & S & S \\
\hline $\mathbf{2 4}$ & $\mathbf{2 3}$ & $\mathbf{3 2}$ \\
\hline $\mathbf{6 6 , 6 6 \%}$ & $\mathbf{6 3 , 8 8 \%}$ & $\mathbf{8 8 , 8 8} \%$ \\
\hline
\end{tabular}

¿La empresa propietaria del software proporciona recursos preconfigurados (bases de datos,

¿La empresa prop
OPACs, etc.)?

¿La base de datos es actualizada periódicamente por la empresa propietaria del producto?

¿La base de datos puede ampliarse indefinidamente en función del número de recursos

disponibles?

TOTAL DE PUNTOS

\begin{tabular}{|r|c|c|c|c|c}
\hline DE LOS CRITERIOS CUMPLIDOS & $16,66 \%$ & $100 \%$ & $83,33 \%$ & $66,66 \%$ & 12 \\
\hline
\end{tabular}

\section{PERSONALIZACIÓN DEL SISTEMA}

¿Se pueden definir conjuntos de recursos para grupos de usuarios distintos?

¿Los campos que se muestran por defecto pueden ser modificados?

¿El listado de resultados que se muestra por defecto puede ser modificado?

¿Se puede personalizar el diseño de la interfaz del usuario?

¿Se pueden crear distintas interfaces para grupos de usuarios distintos?

¿Se pueden definir distintos niveles de acceso según los perfiles de usuarios?

¿Los resultados se pueden enlazar a servicios de entrega de documentos?

¿Se puede añadir el nombre del centro, logos, imágenes y enlaces a la interfaz del producto?

\begin{tabular}{|c|c|c|c|c|}
\hline $\mathrm{N}$ & $S$ & $S$ & $S$ & $S$ \\
\hline $\mathrm{N}$ & $\mathrm{N}$ & $\mathrm{N}$ & $S$ & $\mathrm{~N}$ \\
\hline$S$ & $S$ & $S$ & $S$ & $F$ \\
\hline D & $S$ & $S$ & $S$ & $\mathrm{~N}$ \\
\hline$S$ & $S$ & $S$ & $S$ & $S$ \\
\hline 8 & 16 & 16 & 20 & 9 \\
\hline $40 \%$ & $80 \%$ & $80 \%$ & $100 \%$ & $45 \%$ \\
\hline$P$ & $S$ & $S$ & $S$ & $S$ \\
\hline$D$ & $S$ & $P$ & $\mathrm{~N}$ & $S$ \\
\hline D & $S$ & $S$ & $S$ & $S$ \\
\hline 2 & 12 & 10 & 8 & 12 \\
\hline $16,66 \%$ & $100 \%$ & $83,33 \%$ & $66,66 \%$ & $100 \%$ \\
\hline S & $S$ & $S$ & $S$ & $S$ \\
\hline$S$ & $S$ & $S$ & $S$ & $S$ \\
\hline$S$ & $S$ & $S$ & $S$ & $S$ \\
\hline$S$ & $S$ & $S$ & $S$ & $S$ \\
\hline$S$ & $S$ & $S$ & $S$ & $S$ \\
\hline$S$ & $S$ & $S$ & $S$ & $S$ \\
\hline$S$ & $S$ & $S$ & $\mathrm{~F}$ & $P$ \\
\hline$S$ & $S$ & $S$ & $S$ & $S$ \\
\hline
\end{tabular}




\begin{tabular}{|c|c|c|c|c|c|}
\hline ¿Se puede modificar el esquema de colores que se proporciona de serie? & S & S & S & S & S \\
\hline ¿Las opciones de descarga e impresión se localizan rápidamente y son fáciles de utilizar? & S & S & S & S & S \\
\hline ¿Las opciones de ayuda están disponibles en todas la páginas? & S & S & S & S & S \\
\hline $\begin{array}{l}\text { ¿El sistema permite interrumpir una búsqueda potencialmente demasiado grande y } \\
\text { proporcionarle opciones al usuario para concretar la consulta? }\end{array}$ & S & S & $\mathrm{N}$ & S & S \\
\hline TOTAL DE PUNTOS & 48 & 48 & 44 & 45 & 46 \\
\hline \% DE LOS CRITERIOS CUMPLIDOS & $100 \%$ & $100 \%$ & $91,66 \%$ & $93,75 \%$ & $95,83 \%$ \\
\hline \multicolumn{6}{|l|}{ INTEGRACIÓN CON EL SGB } \\
\hline ¿Permite la integración entre el portal y el SGB local? & $\mathrm{S}$ & $\mathrm{S}$ & $\mathrm{S}$ & S & S \\
\hline ¿Se proporciona información de fondos locales? & $\mathrm{S}$ & $\mathrm{S}$ & $\mathrm{S}$ & $\mathrm{P}$ & $\mathrm{S}$ \\
\hline ¿Permite la integración entre el portal y SGB externos? & $\mathrm{S}$ & S & $\mathrm{S}$ & $\mathrm{S}$ & $\mathrm{S}$ \\
\hline TOTAL DE PUNTOS & 12 & 12 & 12 & 10 & 12 \\
\hline \% DE LOS CRITERIOS CUMPLIDOS & $100 \%$ & $100 \%$ & $100 \%$ & $83,33 \%$ & $100 \%$ \\
\hline \multicolumn{6}{|l|}{ ENTREGA DEL DOCUMENTO FINAL } \\
\hline ¿Permite descargar los documentos finales en el disco duro del ordenador del usuario? & $\mathrm{S}$ & $\mathrm{S}$ & $\mathrm{S}$ & $\mathrm{S}$ & $\mathrm{S}$ \\
\hline ¿Permite enviar los documentos finales por correo electrónico? & $\mathrm{S}$ & $\mathrm{S}$ & $\mathrm{S}$ & S & $\mathrm{S}$ \\
\hline TOTAL DE PUNTOS & 8 & 8 & 8 & 8 & 8 \\
\hline \% DE LOS CRITERIOS CUMPLIDOS & $100 \%$ & $100 \%$ & $100 \%$ & $100 \%$ & $100 \%$ \\
\hline \multicolumn{6}{|l|}{ ESTADISTTICAS } \\
\hline ¿Se pueden obtener registros de transacciones (logs) y estadísticas de uso? & S & $\mathrm{S}$ & $\mathrm{S}$ & $\mathrm{S}$ & $\mathrm{S}$ \\
\hline TOTAL DE PUNTOS & 4 & 4 & 4 & 4 & 4 \\
\hline \% DE LOS CRITERIOS CUMPLIDOS & $100 \%$ & $100 \%$ & $100 \%$ & $100 \%$ & $100 \%$ \\
\hline \multicolumn{6}{|l|}{ SEVIDOR DE ENLACES } \\
\hline ¿Incorpora un servidor de enlaces? & S & $\mathrm{S}$ & $\mathrm{N}$ & $\mathrm{S}$ & $\mathrm{S}$ \\
\hline TOTAL DE PUNTOS & 4 & 4 & 0 & 4 & 4 \\
\hline \% DE LOS CRITERIOS CUMPLIDOS & $100 \%$ & $100 \%$ & $0 \%$ & $100 \%$ & $100 \%$ \\
\hline \multicolumn{6}{|l|}{ ENTORNOS CONSORCIADOS } \\
\hline ¿Está diseñado para gestionar entornos consorciados? & $\mathrm{P}$ & $\mathrm{S}$ & $\mathrm{P}$ & $\mathrm{P}$ & $\mathrm{P}$ \\
\hline TOTAL DE PUNTOS & 2 & 4 & 2 & 2 & 2 \\
\hline \% DE LOS CRITERIOS CUMPLIDOS & $50 \%$ & $100 \%$ & $50 \%$ & $50 \%$ & $100 \%$ \\
\hline \multicolumn{6}{|l|}{ SOPORTE POST-VENTA } \\
\hline ¿La empresa o sus distribuidores realizan la instalación y proporcionan soporte inicial? & $S$ & S & $S$ & $S$ & $S$ \\
\hline ¿Se proporciona el software a las bibliotecas para que realicen la instalación por cuenta propia? & $\mathrm{N}$ & $\mathrm{N}$ & S & $\mathrm{N}$ & S \\
\hline ¿El precio incluye toda la información relacionada con el producto? & S & S & S & S & S \\
\hline ¿Se proporciona formación onsite? & S & S & S & S & S \\
\hline $\begin{array}{l}\text { ¿Existen listas de distribución, grupos de trabajo y grupos de usuarios en España de estos } \\
\text { productos? }\end{array}$ & $\mathrm{N}$ & S & $\mathrm{N}$ & $\mathrm{N}$ & $\mathrm{N}$ \\
\hline ¿Dispone de oficina o distribuidor en España? & $\mathrm{N}$ & S & S & S & S \\
\hline Otro tipo de soporte & S & S & S & S & S \\
\hline TOTAL DE PUNTOS & 16 & 24 & 24 & 20 & 24 \\
\hline$\%$ DE LOS CRITERIOS CUMPLIDOS & $57,14 \%$ & $85,71 \%$ & $85,71 \%$ & $71,43 \%$ & $85,71 \%$ \\
\hline TOTAL DE PUNTOS & 268 & 300 & 267 & 280 & 294 \\
\hline$\%$ DE LOS CRITERIOS CUMPLIDOS & $82,72 \%$ & $92,59 \%$ & $82,41 \%$ & $86,42 \%$ & $90,74 \%$ \\
\hline Leyenda & \multicolumn{5}{|c|}{ Tabla } \\
\hline \multicolumn{6}{|l|}{ S: Si. El producto cumple el requisito (4ptos). } \\
\hline \multicolumn{6}{|l|}{$\mathbf{N}$ : No. El producto no cumple el requisito (0 ptos). } \\
\hline \multicolumn{6}{|l|}{ D: Desconocido. El productor no proporciona información al respecto (0 ptos). } \\
\hline \multicolumn{6}{|l|}{ P: Parcialmente. El producto cumple el requisito parcialmente (2 ptos). } \\
\hline F: Futuro. El producto cumplirá el requisito en la próxima versión (1 pto). & & & & & \\
\hline
\end{tabular}

var a cabo metaconsultas: cómo se traducen las consultas, cómo se interrogan los recursos, qué formatos se utilizan y con qué protocolos de intercambio trabajan. También hay que valorar el número de recursos que se pueden consultar conjuntamente así como la velocidad en la respuesta.

c. Conectividad. Una de las tareas más largas y costosas es la integración de los recursos contratados en el portal. La manera en que se haga este proceso y cómo se mantenga es fundamental para el buen funcionamiento del producto. Algunas soluciones vienen con una base de datos de recursos predefinidos que agilizan el procedimiento de alta; habrá que valorar su número y su relevancia, la escalabilidad de la base de datos y el mantenimiento, que en función del producto irá a cargo de la biblioteca o del vendedor. d. Desduplicación de resultados. La capacidad de eliminar resultados duplicados la ofrecen la mayoría de productos. Es interesante evaluar la forma cómo se muestran y la manera de informar al usuario de la duplicidad del documento solicitado.

3. Servicios de personalización. Hace referencia a todos aquellos servicios que permiten al usuario establecer sus preferencias de visualización y búsqueda, almacenar consultas, activar alertas, crear listados personalizados tipo "MyJournals" o "MyDatabases", etc.

4. Autentificación. La capacidad del sistema para diferenciar entre usuarios registrados e invitados. Esta función es especialmente necesaria para poder garantizar el uso correcto de las licencias de las bases de datos o revistas electrónicas contratadas. 
5. Gestión de copyright. Posibilidad de gestionar los derechos de propiedad intelectual de los objetos y colecciones digitales de la institución.

6. Gestión de los recursos electrónicos. Capacidad de controlar de manera efectiva el ciclo de vida de dichos recursos: selección, adquisición, evaluación, mantenimiento, renovación y cancelación.

7. Integración con el SGB. Potencial para consultar los fondos de la biblioteca a través del portal, así como consultar catálogos de otros centros. También hay que valorar la capacidad de ofrecer servicios habitualmente suministrados a través del opac: peticiones de préstamos interbibliotecarios, reservas, localización de fondos, etc.

8. Servicios de enlaces. Capacidad de revisión de los vínculos ofrecidos en el portal, así como la posibilidad de disponer de un servidor de enlaces OpenURL que ofrezca servicios sensibles al contexto de búsqueda del usuario.

9. Entrega del documento final. Además de valorar cómo se puede buscar la información y la forma de recuperarla, hay que investigar cómo consigue el usuario los documentos finales. Si bien la mayoría de sistemas permiten que el usuario almacene los resultados en el disco duro del ordenador o los envíe por correo electrónico, otros lo obligan a desplazarse físicamente a la biblioteca para recogerlos.

10. Estadísticas. Es importante que el portal proporcione datos sobre su uso, pudiendo determinar cuáles son los materiales más consultados, los que no se utilizan, aquellos que se solicitan pero que la biblioteca no tiene contratados, etc. También debe permitir segmentar la información por grupos de usuarios para detectar necesidades y preferencias.

11. Precio. Es determinante para elegir el sistema deseado. Las modalidades de precio más comunes son en función del número de usuarios potenciales de la biblioteca, o bien dependen de la cantidad de colecciones disponibles.

Otro aspecto que se debe valorar es la capacidad de gestión consorciada de recursos. Hay que destacar que no todos los programas están concebidos desde su creación para realizar dicha función, aunque ofrezcan soluciones para trabajar en entornos consorciados. Productos como MetaLib y SFX están especialmente concebidos con esta finalidad, a la vez que aseguran la independencia de cada biblioteca miembro de un consorcio. Así, los medios informativos que adquiere el consorcio se gestionan de manera única para todos los centros desde los servicios centrales, mientras que las bibliotecas tienen plena libertad para organizar y con- trolar aquellos fondos que han contratado de manera individual.

Centrándonos en el ámbito español, los productos disponibles por la presencia de las empresas productoras en el mercado estatal, son los siguientes: MetaLib, EnCompass, Millenium Access Plus, Vectors, Sirsi SingleSearch. En la tabla adjunta mostramos una comparativa de los distintos productos que puede ayudar a conocer mejor las soluciones valorando aspectos muy concretos de su funcionamiento.

Si bien todos los productos superan con nota la mayoría de los criterios evaluados, podemos destacar diferencias considerables en 7 de los aspectos analizados: servicios personalizados, autentificación, protocolos de comunicación, sistema, base de datos de recursos preconfigurados, entornos consorciados y soporte post-venta. Según estos criterios, la solución de Ex Libris es la más completa por cumplir un mayor número de requisitos, aunque es importante valorar las necesidades de los usuarios de cada centro antes de decidirse por un producto en concreto.

En el terreno práctico, las soluciones más escogidas por el mercado universitario y de investigación español son MetaLib y Millenium Access Plus. Tanto el Consejo Superior de Investigaciones Científicas, que cuenta con casi 100 bibliotecas especializadas, como la Universidad Politécnica de Valencia han anunciado la adquisición de MetaLib para gestionar la biblioteca híbrida en sus entornos bibliotecarios. Por su parte, el Consorcio de Bibliotecas Universitarias de Andalucía ha hecho pública la compra de Millenium Access Plus como solución para gestionar los recursos electrónicos contratados, mientras que la biblioteca de la Universidad Complutense de Madrid ya lo tiene instalado como complemento de su SGB.

\section{Conclusión}

Las posibilidades de organización de los catálogos colectivos van más allá de lo que podíamos imaginar hace unos años. La creación de redes bibliotecarias heterogéneas donde colaboran grandes y pequeñas bibliotecas con fondos variables y con sistemas de gestión bibliotecaria diferentes son, hoy por hoy, una realidad.

Los distintos modelos de catálogo colectivo permiten una completa flexibilidad en cuanto a su creación y a las múltiples combinaciones de tipologías bibliotecarias que se pueden hacer. El hecho de encontrar más de un modelo da la oportunidad a los consorcios de trabajar de la manera más cómoda posible según sus preferencias. Como ya hemos visto, algunos prefieren modelos centralizados donde cualquier cambio esté controlado por un organismo central, otros 
prefieren que cada biblioteca sea completamente independiente, mientras que hay quien se conforma con la fusión de registros en el momento de la consulta y la visualización por parte del usuario.

\section{«Los portales bibliotecarios permiten catalogar y controlar revistas electrónicas, bases de datos, e-books, etc., a la vez que proporcionan mecanismos de búsqueda transversal»}

Otro aspecto importante a destacar es el cambio que se ha producido en el mercado del software para bibliotecas y que, inevitablemente, ha modificado o modificará su manera de trabajar.

Los desarrollos informáticos que han llevado a cabo los productores de software bibliotecario ( $E x \mathrm{Li}^{-}$ bris, Endeavor, VTLS, Innovative Interfaces, etc.) han permitido integrar fondos físicos y electrónicos combinando necesidades tan distintas como las que suscitan la gestión de estos tipos de recursos.

La evolución de los productos bibliotecarios comerciales en los últimos tres años ha sido tan grande y tan importante que ha abierto las puertas a nuevas posibilidades de organización y de crecimiento en las bibliotecas. La consolidación de estos sistemas en los próximos tres o cuatro años y la aparición de nuevos productos para gestionar colecciones multimedia, cambiarán el rumbo de las bibliotecas, por dos razones principales:

-En primer lugar, porque supondrán un avance en la gestión interna de los fondos electrónicos (a nivel de catalogación, organización y control administrativo). Los sistemas que actualmente se utilizan para gestionar los recursos electrónicos (especialmente en bibliotecas universitarias) siguen el modelo tradicional de gestión de los recursos físicos sin tener en cuenta las características propias de los electrónicos. Además, son soluciones parciales y temporales que sólo cubren determinados aspectos de esta gestión dificultando enormemente el intercambio de información y la gestión cooperativa o consorciada.

-Por otra parte, supondrán un cambio radical en cuanto a la presentación de las colecciones al usuario. El punto de partida inicial para la búsqueda de información pasará a ser el portal bibliotecario adquiriendo el rol que hasta el momento ha tenido el web opac. Al contrario de lo que pueda parecer, esto no implicará un menor uso del opac sino que proporcionará una plataforma de acceso único a todos los recursos y potenciará el uso de éstos aumentando su visibilidad.
Además, estos productos serán clave para la gestión consorciada de colecciones puesto que proporcionan instrumentos necesarios para permitir un acceso regulado a las obras, especialmente a las electrónicas.

Habrá que estudiar a fondo los cambios que se van a producir en los próximos años y que van a afectar claramente tanto a los consorcios como a las bibliotecas individuales. Sin duda estamos en un momento importantísimo de la gestión de las colecciones en entornos consorciados en el cual los productos de los que hemos hablado y los distintos modelos de constitución de catálogos colectivos estarán en el punto de mira principal de los profesionales de la biblioteconomía y la documentación.

\section{Notas}

1. Catálogo Melvyl actual:

http://www.melvyl.cdlib.org

2. La California Digital Library es la $11^{\mathrm{a}}$ universidad que forma parte de la University of California.

3. Catálogo $C U N Y$ actual:

http://apps.appl.cuny.edu: $83 / F$

4. Catálogo Rebiun actual:

http://www.crue.org/cgi-bin/rebiun

5. Catálogo actual de la Austrian Library Network:

http://magnum.bibvb.ac.at/

6. Catálogo actual del $C B U C$ :

http://www.cbuc.es/ccuc

7. LinkSolver no es un portal sino un gestor de enlaces.

\section{Referencias bibliográficas}

Anglada, Lluís M. "Cooperació bibliotecària a Espanya”. En: Item, 1995, gener-juny, v. 16, pp. 51-67.

Anglada, Lluís M. "Impacte i influència dels consorcis en la gestió de les col-leccions". En: BiD: textos universitaris de biblioteconomia i documentació, 2003, juny, n. 10. Consultado en: 09-12-04.

http://www2.ub.es/bid/consulta_articulos.php?fichero=10anglada.htm

Anglada, Lluís M.; Comellas, Núria. "Què és just?: models de preus a l'era electrònica". En: BiD: textos universitaris de biblioteconomia $i$ documentació, 2002, juny, n. 8. Consultado en: 09-12-04.

http://www.ub.es/biblio/bid/08angla1.htm

Cabo, Mercè, [et al.]. "El puente hacia el futuro: el plan estratégico de Rebiun". En: BiD: textos universitaris de biblioteconomia $i$ documentació, 2003, juny, n. 10. Consultado en: 05-01-05. http://www.ub.es/bid/10cabo2.htm

Chvatal, Don. “ALA 2003 Midwinter report”. En: Biblio tech review, [2003]. Consultado en: 11-04-05.

http://www.biblio-tech.com/btr11/ALA_2003_MW_Complete.cfm

Dorner, Daniel G.; Curtis, Anne Marie. A comparative review of common user interface software products for libraries. Wellington: Victoria University of Wellington, 2003. Consultado en: 11-04-05. http://www.natlib.govt.nz/files/CUI_Report_Final.pdf

Ferguson, Christine L.; Grogg, Jill E. "The helping you buy series: comparing competing library technology products: your guide to vendor product facts: October: openURL link resolvers". En: Computers in libraries, 2004, October, pp. 17-24.

Giordano, Tommaso. "Library consortium models in Europe: a comparative analysis". En: Alexandria, 2002, v. 14, n. 1, pp. 41-52.

Jacsó, Péter. "Thoughts about federated searching". En: Information today, 2004, October, v. 21, n. 9, pp. 17-40. 


\title{
Ahora renovar (o comenzar) la suscripción a "El profesional de la información” es mucho más ágil y sencillo.
}

\author{
Usted puede gestionar online su suscripción conectándose a \\ esta página web:
}

http://www.elprofesionaldelainformacion.com/suscripciones.htmI

\author{
Si lo desea puede comunicar con nosotros dirigiéndose a es- \\ ta dirección de correo electrónico:
}

\author{
suscripciones@elprofesionaldelainformacion.com
}

Kaplan, Michael. "Serials aggregations-multiple versions and the virtual union catalog: the California Digital Library, SUNY, and Ex Libris experiences". En: North American Serials Interest Group, 2003. Consultado en: $10-12-04$

http://www.exlibrisgroup.com/aleph_articles.htm

Lewis, Nicholas. "Talking about a revolution? First impressions of Ex Libris's MetaLib”. En: Ariadne, 2002, June-July, n. 32. Consultado en: 0312-04.

http://www.ariadne.ac.uk/issue32/metalib

Merlo Vega, José Antonio. "La cooperación en las bibliotecas universitarias: fundamentos y redes cooperativas". En: Boletín de la Asociación Andaluza de Bibliotecarios, 1999, n. 54, pp. 33-57.

Merlo Vega, José Antonio; Sorli Rojo, Ángela. "Las bibliotecas como clientes y servidoras de información web". En: 6es. Jornades Catalanes de Documentació. Barcelona: Col-legi Oficial de Bibliotecaris-Documentalistes de Catalunya, 1997, pp. 317-327.

Merlo Vega, José Antonio. "Cooperación bibliotecaria en Europa: programas y proyectos". En: Artículos. Facultad de Traducción y Documentación. Universidad de Salamanca, 1998. Consultado en: 03-01-05. http://exlibris.usal.es/merlo/escritos/toledo.htm

Merlo Vega, José Antonio; Sorli Rojo, Ángela. "La cooperación bibliotecaria en tiempos de internet". En: Anuari Socadi de documentació $i$ informació. Barcelona: Societat Catalana de Documentació i Informació, 1997-1998, pp. 245-254.

Ramírez Escárcega, Alejandro. "Conclusiones del segundo seminario de cooperación en bibliotecas y centros de información". En: Memorias XV Jornadas mexicanas de biblioteconomía. México: Ambac, 1984, p. 187.

Ruiz Rivera, Leticia. "La cooperación bibliotecaria: un reto a vencer". En: IV Encuentro latinoamericano de bibliotecarios teológicos, 2002, pp. 1-11. Consultado en: 01-12-04.

http://www.ibiblio.org/rlit/noticias/encuent4/Leticia_La_Coop.pdf

Sales Zaguirre, Jordi. Models cooperatius d'assignació de costos en un consorci de biblioteques. Barcelona: Universitat de Barcelona, 2002. Isbn 84-688-1742-2. Consultado en: 01-12-04.
http://www.tdx.cesca.es/TDX-0318103-161333/index_an.html

Sadeh, Tamar. "The callenge of metasearching". En: New library world, 2004, v. 105, n. 1196/1997, pp. 69-75.

Sadeh, Tamar. "To Google or not to Google: metasearch design in the quest for the ideal user experience". En: ELAG: interoperability, new challenges and solutions, 28th library systems semina, 2004. Consultado en 25-11-04.

http://www.elag2004.no

Sadeh, Tamar. "Integrated access to hybrid information resources". En: Liber quarterly, 2003, v. 13, n. 1-4, pp. 299-311.

Sadeh, Tamar; Walker, Jenny. "Library portals: toward the semantic web". En: New library world, 2003, v. 104, n. 1/2, pp. 11-19.

Sadeh, Tamar. "MetaLib and SFX: managing heterogeneous resources in the scholarly environment". En: Caslin: Library Academy of Sciences of Czech Republic and National Library of the Czech Republic, 2001. Consultado en: 03-12-04.

http://www.caslin.cz:7777/caslin01/sbornik/metalib.html

Sorli Rojo, Ángela; Merlo Vega, José Antonio. "Catálogos colectivos de bibliotecas españolas en internet". En: Revista española de documentación científica, 2002, julio-septiembre, v. 25, n. 3, pp. 321-328.

Stubbings, Ruth; Hamblin, Yvonne. "How to make your e-resources earn their keep”. En: Serials, 2004, March, v. 17, n. 1, pp. 25-30.

Voigt, Melvin, (edit.). Advances in librarianship, 1977, v. 7, pp. 173.

Welter, Jacqueline W. "Library cooperation: wave of the future or ripple?”. En: Bulletin of the Medical Library Association, 1975, January, v. 63, n. 1, pp. 1-6.

Gisela Ruiz Chacón.

giseleta@gmail.com 